\title{
Nur keinen Stress
}

- Was ist der größte Stressfaktor im beruflichen Alltag von Ergotherapeuten? Diese Frage stellten wir uns in der Redaktion, als wir für einen Cartoonisten typische Ergotherapie-Stressoren sammelten. Jede von uns konnte eine Anekdote aus der Praxis zum Besten geben: Termine organisieren, Verordnungen korrigieren lassen, nach Hausbesuchen pünktlich zur nächsten Therapie in die Praxis zurückkehren, den Erwartungen von Klienten gerecht werden... Ihnen fallen sicher weitere Stressoren ein.

_ Aber was tun, wenn es nicht beim kurzfristigen Stress bleibt, sondern man zunehmend merkt, dass Stress und Zeitdruck überhandnehmen? Wie erkennt man, wenn es zu viel wird und man „ausbrennt"? Und wie schafft man es, dass es gar nicht erst so weit kommt? Sabine Josten ist in ihrem Beitrag zum Thema Burn-out diesen Fragen nachgegangen (๑ Seite 36).

_ Auch wenn wir einen helfenden Beruf gewählt haben: Für eine erfolgreiche Therapie sind auch unsere Klienten mitverantwortlich. Wir müssen sie über den gesamten Therapieprozess ins Boot holen, denn gemeinsam paddelt es sich schneller und leichter. Wie gelingt es uns allerdings, die Klienten auch in ihrem Alltag zum Paddeln zu motivieren? Der Artikel „Alltagsnahe Ergotherapie“ gibt Anregungen, wie dies in der Pädiatrie klappen kann (๑ Seite 20).

- Verzögert sich der Therapiebeginn oder schließt man eine Einheit zu spät $a b$, gerät häufig der komplette Zeitplan ins Wanken - ebenfalls eine Stresssituation. Passiert das öfter, sind die wartenden Klienten verärgert. Wie Sie souverän mit deren Beschwerden umgehen, lesen Sie im Artikel von Ulla Beushausen ( $\odot$ Seite 30).

Eine entspannte Lektüre, und geben Sie gut auf sich Acht, Ihre

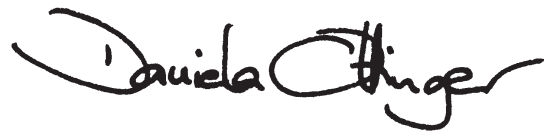

\section{INTERNET}

\section{Fachwissen für} Ergotherapeuten

Sie interessieren sich für ergotherapeutische Fachbücher, Zeitschriften und Veranstaltungen? Dann schauen Sie doch mal im Internet unter www.thieme.de/ergoonline. Dort finden Sie zudem Assessments zum Downloaden und nützliche www-Tipps. 